\title{
TWO NEW OPEN MAPPING THEOREMS FOR ANALYTIC MULTIVALUED FUNCTIONS
}

\author{
LINE BARIBEAU AND SARAH HARBOTTLE
}

(Communicated by Clifford J. Earle, Jr.)

\begin{abstract}
It is conjectured that the image $U$ of a domain $D$, under an analytic multivalued function $K$, satisfies $U \cap \partial U \subset \bigcap_{\lambda \in D} K(\lambda)$ if the values of $K$ are polynomially convex sets. We prove that this conjecture is true in the two special cases when $K$ is convex-valued and polar-valued.
\end{abstract}

This paper is concerned with the extension of the classical open mapping theorem for holomorphic functions to the more general setting of analytic multivalued (a.m.v.) functions. This problem was first considered by T. J. Ransford in [R1] where, although special cases are proved, examples are given that preclude a general theorem. However the counterexamples given there leave open the following

Conjecture. Let $D$ be a domain and $K$ an a.m.v. function defined on $D$ whose values are polynomially convex sets. Denote by $U$ the image of $D$, i.e. $U=$ $\bigcup_{\lambda \in D} K(\lambda)$. Then we have $U \cap \partial U \subset \bigcap_{\lambda \in D} K(\lambda)$.

In particular this would imply that $U$ is open whenever $\bigcap_{\lambda \in D} K(\lambda)$ is empty. In this note we shall prove that this conjecture is true in two special cases. In the first theorem $K(\lambda)$ will be a convex set, while in the second $K(\lambda)$ will be a polar set. It is interesting to note that these represent extreme examples of polynomially convex sets in the sense that convex sets are rather "fat," while polar sets are very "thin."

Before stating our theorems, let us recall the relevant definitions. Here $\kappa(\mathbb{C})$ denotes the set of nonempty compact subsets of $\mathbb{C}$, and $D$ a domain in the complex plane. We say that a multivalued function defined on a topological space $X$ is upper semicontinuous (u.s.c.) if for every open set $V$, the set $\{\lambda \in X: K(\lambda) \subset V\}$ is open in $X$.

Definition. A multivalued function $K: D \rightarrow \kappa(\mathbb{C})$ is analytic if it satisfies the following conditions:

(1) $K$ is upper semicontinuous.

(2) Whenever $D_{1}$ is an open subset of $D, V$ an open neighbourhood of $\operatorname{Gr}\left(\left.K\right|_{D_{1}}\right)=\left\{(\lambda, z): \lambda \in D_{1}, z \in K(\lambda)\right\}$ and $\psi(\lambda, z)$ a plurisubhar-

Received by the editors January 11, 1991.

1980 Mathematics Subject Classification (1985 Revision). Primary 32F05; Secondary 32E20, $30 \mathrm{G} 30$. 
monic function on $V$, then the function

$$
\Psi(\lambda)=\sup \{\psi(\lambda, z): z \in K(\lambda)\}
$$

is subharmonic on $D_{1}$.

An alternative definition is provided by the following deep result:

Theorem [Sł]. The function $K: D \rightarrow \kappa(\mathbb{C})$ is analytic if and only if it is upper semicontinuous and the open set $\{(\lambda, z): \lambda \in D, z \notin K(\lambda)\}$ is pseudoconvex.

A classical example of an a.m.v. function is the spectrum of an analytic family of elements of a Banach algebra. Applications to functional analysis can be found for instance in $[\mathrm{A}]$ or $[\mathrm{St}]$.

As for the classical version, the proof of our first open mapping theorem will be based on the following multivalued analogue of Rouchés Theorem.

Theorem [R2]. Suppose $(\lambda, t) \mapsto K_{t}(\lambda): \bar{D} \times[0,1] \rightarrow \kappa(\mathbb{C})$ is upper semicontinuous and that

(1) for each fixed $t \in[0,1], K_{t}$ is analytic on $D$,

(2) for each fixed $\lambda \in D, K_{t}(\lambda)$ is continuous on $[0,1]$.

Then

$$
K_{0}(D) \triangle K_{1}(D) \subset \bigcup_{\substack{\zeta \in \partial D \\ t \in[0,1]}} K_{t}(\zeta) .
$$

Consider a multivalued function $K$ analytic on the unit disc $\Delta$, u.s.c. on $\bar{\Delta}$, and such that $K(\zeta)$ is convex for all $\zeta \in \partial \Delta$. It follows from the proof of Theorem 1 of [B-R] that for any fixed $z_{0} \in K(0)$, one can construct a function $h \in H^{\infty}(\Delta)$ such that $h(0)=z_{0}$ and, for all $\zeta \in \partial \Delta$,

$$
\operatorname{dist}(h(\lambda), K(\zeta)) \rightarrow 0 \quad \text { as } \lambda \rightarrow \zeta, \lambda \in \Delta .
$$

We shall make this into a multivalued function by defining $H(\lambda)=\{h(\lambda)\}$ on $\Delta$ and $H(\zeta)(\zeta \in \partial \Delta)$ as the cluster set of $h$ at $\zeta$, i.e.

$$
H(\zeta)=\left\{z: \exists \lambda_{n} \in \Delta, \lambda_{n} \rightarrow \zeta, h\left(\lambda_{n}\right) \rightarrow z\right\} .
$$

The resulting multivalued function is analytic on $\Delta$, u.s.c. on $\bar{\Delta}$ and satisfies $H(\zeta) \subset K(\zeta)$ for all $\zeta \in \partial \Delta$.

Theorem 1. Let $K: D \rightarrow \kappa(\mathbb{C})$ be an a.m.v. function whose values are convex sets. Then $U \cap \partial U \subset \bigcap_{\lambda \in D} K(\lambda)$.

Proof. Let $z_{0} \in U \cap \partial U$. We will show that for any $\lambda_{0}$ such that $z_{0} \in K\left(\lambda_{0}\right)$ we have $z_{0} \in K\left(\lambda_{0}+r e^{i \theta}\right)$ for all $\theta \in[0,2 \pi]$ and $r$ small enough. It will then follow by upper semicontinuity and connectedness that $z_{0} \in \bigcap_{\lambda \in D} K(\lambda)$.

Let $r$ be such that $\left\{\lambda:\left|\lambda-\lambda_{0}\right| \leq r\right\} \subset D$. Without loss of generality, we may assume that $\lambda_{0}=0$ and $r=1$. For $\lambda \in \Delta$ and $t \in[0,1]$, let

$$
K_{t}(\lambda)=t K(\lambda)+(1-t) H(\lambda)
$$

where $H(\lambda)$ is defined as above. Applying Rouché's theorem we get

$$
K(\Delta) \Delta H(\Delta) \subset \bigcup_{\substack{\zeta \in \partial \Delta \\ t \in[0,1]}} K_{t}(\zeta)=\bigcup_{\zeta \in \partial \Delta} K(\zeta),
$$


where the last equality follows from the convexity of $K$ and $H \subset K$. If $h$ were not constant, then $H(\Delta)$ would contain an open neighbourhood of $z_{0}$, so it would contain points of $\mathbb{C} \backslash K(D)$, contradicting (1). So $h \equiv z_{0}$ and $z_{0} \in K(\zeta)$ for all $\zeta \in \partial \Delta$.

The proof of our second theorem rests on the following result of A. Sadullaev. Theorem [Sa]. Suppose $K: D \rightarrow \kappa(\mathbb{C})$ is an a.m.v. function whose values are polar sets. Then there is a function $\psi: D \times \mathbb{C} \rightarrow[-\infty, \infty)$ which is plurisubharmonic on $D \times \mathbb{C}$ and pluriharmonic on $D \times \mathbb{C} \backslash \operatorname{Gr}(K)$ and is such that $\psi \equiv-\infty$ on $\operatorname{Gr}(K)$.

For an u.s.c. function $K: D \rightarrow \kappa(\mathbb{C})$ and $z_{0} \in U$ we define

$$
L\left(z_{0}\right)=\left\{\lambda \in D: z_{0} \in K(\lambda)\right\}
$$

and note that $L\left(z_{0}\right)$ is a closed set. We then have

Theorem [R1]. Suppose that for some $z_{0} \in U$ there is an open set $D_{1} \subset D$ such that

$$
\varnothing \neq L\left(z_{0}\right) \cap D_{1} \subset \subset D_{1} .
$$

Then $z_{0} \in$ int $U$.

Theorem 2. Let $K: D \rightarrow \kappa(\mathbb{C})$ be an a.m.v. function whose values are polar sets. Then $U \cap \partial U \subset \bigcap_{\lambda \in D} K(\lambda)$.

Proof. Let $\psi$ be the plurisubharmonic function given by Sadullaev's theorem. For $z_{0} \in U$,

$$
L\left(z_{0}\right)=\left\{\lambda: \psi\left(\lambda, z_{0}\right)=-\infty\right\},
$$

and so, since $\psi\left(\lambda, z_{0}\right)$ is a subharmonic function of $\lambda$, the set $L\left(z_{0}\right)$ is polar or is the whole of $D$. We choose $z_{0} \in U \cap \partial U$, and suppose for a contradiction that $L\left(z_{0}\right)$ is polar. We can find $r>0$ such that

$$
\left\{\lambda:\left|\lambda-\lambda_{0}\right|=r\right\} \cap L\left(z_{0}\right)=\varnothing .
$$

Denoting $\left\{\lambda:\left|\lambda-\lambda_{0}\right|<r\right\}$ by $D_{1}$, we then have

$$
\varnothing \neq L\left(z_{0}\right) \cap D_{1} \subset \subset D_{1},
$$

and so conclude that $z_{0} \in$ int $U$, a contradiction. Thus $L\left(z_{0}\right)=D$, or equivalently $z_{0} \in \cap_{\lambda \in D} K(\lambda)$.

\section{ACKNOWLEDGMENTS}

The authors wish to thank T. J. Ransford for helpful discussions, and N. Méda who translated Sadullaev's paper. This work was supported in parts by NSERC (Canada) and SERC (U.K.).

\section{REFERENCES}

[A] B. Aupetit, Analytic multivalued functions in Banach algebras and uniform algebras, Adv. in Math. 44 (1982), 18-60.

[B-R] B. Berndtsson and T. J. Ransford, Analytic multifunctions, the $\bar{\partial}$-equation, and a proof of the corona theorem, Pacific J. Math. 124 (1986), 57-72. 
[R1] T. J. Ransford, Open mapping, inversion and implicit function theorems for analytic multivalued functions, Proc. London Math. Soc. (3) 49 (1984), 537-562.

[R2] _ On the range of an analytic multivalued function, Pacific J. Math. 123 (1986), 421-439.

[Sa] A. Sadullaev, Pseudoconcave sets and algebraic lemniscates, preprint.

[Sł] Z. Slodkowski, Analytic set-valued functions and spectra, Math. Ann. 256 (1981), 363-386.

Department of Pure Mathematics and Mathematical Statistics, Universi ty of Cambridge, 16 Mill LaNe, CAMbridge CB2 1SB, United Kingdom

E-mail address, Line Baribeau: LB110@phoenix.cambridge.ac.uk 